\title{
ON THE REPRESENTATION OF A FUNCTION BY A SERIES OF LEGENDRE'S FUNCTIONS
}

\author{
By E. W. HoBson.
}

[Received October 4th, 1908.-Read November 12th, 1908.]

In my paper* "On a General Convergence Theorem and the Theory of the Representation of a Function by Series of Normal Functions" I considered the convergence of the series of Legendre's functions which represents a function $f(x)$ defined for the interval $(-1,1)$ of the real variable $x$. It was there shewn that, if $f(x)$ have a Lebesgue integral in the interval $(-1,1)$, then the series converges to the value

$$
\frac{1}{2}\{f(x+0)+f(x-0)\}
$$

at any interior point $x$ of the interval $(-1,1)$, for which a neighbourhood exists in which $f(x)$ is of limited total fluctuation (à variation bornée) provided the sufficient condition be satisfied, that there exist neighbourhoods of the end-points $-1,1$, in which $f(x)$ is of limited total fluctuation. The chief object of the present communication is to shew that this last condition may be replaced by the less stringent one, that it is sufficient that $\frac{f(x)}{\left(1-x^{2}\right)^{3}}$ have a Lebesgue integral in the interval $(-1,1)$. This is equivalent to the condition that both $\frac{f(x)}{(1-x)^{\frac{1}{2}}}$ and $\frac{f(x)}{(1+x)^{\frac{1}{2}}}$ should have Lebesgue integrals in $(-1,1)$. The condition is satisfied, in particular if $|f(x)|$ have finite upper limits in neighbourhoods of the points $-1,1$; and more generally when those points are points of infinite discontinuity of $f(x)$, of types limited by the necessity for the convergence of the integrals of $\frac{f(x)}{\left(1-x^{2}\right)^{2}}$. The difficulty in connection with the end-points of the interval arises from the fact that the asymptotic expressions for the Legendre's functions are applicable only for values of $x$ in an interval $(-1+\epsilon, 1-\epsilon)$, and not for all values of $x$ in the interval $(-1,1)$. This difficulty is here surmounted by the establish- 
ment of the theorem that $n^{\frac{1}{2}\left(1-x^{2}\right)^{\ddagger}} P_{n}(x)$ is numerically less than a fixed positive number, independent of $n$ and $x$, for all (integral) values of $n$, and for all values of $x$ in the interval $(-1,1)$.

In the latter part of this paper, the question of the convergence of the series at the end-points of the interval $(-1,1)$ is treated in some detail. It is shewn that, for such convergence, much more stringent conditions as regards the nature of the function $f(x)$ are requisite than for the convergence of the series at an interior point of the interval. This investigation may prove of interest as exhibiting the effect of the singularities of a differential equation, in this case Legendre's equation, upon the series of normal functions.

1. It is known that, in any interval $(\eta, \pi-\eta)$, where $\eta$ is an arbitrarily small positive number, $P_{n}(\cos \theta)$ is represented by

$$
\left.\left(\frac{2}{n \pi \sin \theta}\right)^{\frac{1}{2}}\left[\cos \left(n+\frac{1}{2}\right) \theta-\frac{\pi}{4}\right\}+\frac{\alpha(n, \theta)}{n}\right],
$$

where $\alpha(n, \theta)$ is, for all values of $n$ and $\theta$, numerically less than some tixed positive number. It is, however, necessary for the purpose of estimating the limiting value of an integral taken through the neighbourhood of either of the points $\theta=0, \theta=\pi$, when the integral involves $P_{n}(\cos \theta)$, to have information as to the mode in which $P_{n}(\cos \theta)$ varies with $n$ and $\theta$ for all integral values of $n$ and for all values of $\theta$ in the interval $(0, \pi)$. It will will here be proved that $(n \sin \theta)^{\frac{1}{1}} P_{n}(\cos \theta)$ is numerically less than some tixed positive number for all values of $\theta$ in the interval $(0, \pi)$, and for all positive integral values of $n$.

It is known that $\frac{1}{4} \pi P_{n}(\cos \theta)$ is given for every value of $\theta$ such that $0<\theta<\pi$ by the convergent series

$$
\begin{aligned}
\frac{2.4 \ldots 2 n}{3.5 \ldots 2 n+1}[\sin (n+1) \theta & +\frac{1 . n+1}{1.2 n+3} \sin (n+3) \theta \\
& \left.+\frac{1.3}{1.2} \frac{(n+1)(n+2)}{(2 n+3)(2 n+5)} \sin (n+5) \theta+\ldots\right],
\end{aligned}
$$

which may be written in the form

$$
\begin{aligned}
& \Pi\left(\frac{1}{2}\right)\left[\frac{\Pi(n)}{\Pi\left(n+\frac{1}{2}\right)} \sin (n+1) \theta+\frac{1}{2} \frac{\Pi(n+1)}{\Pi\left(n+\frac{3}{2}\right)} \sin (n+3) \theta+\ldots\right. \\
& \left.+\frac{1.3 \ldots 2 r-1}{2.4 \ldots 2 r} \frac{\Pi(n+r)}{\Pi\left(n+r+\frac{1}{2}\right)} \sin (n+\overline{2 r+1}) \theta+\ldots\right] \\
& \text { Since } \quad \Pi(x)=e^{-x} x^{x}(2 \pi x)^{\frac{1}{4}}\left(1+\frac{A}{\sqrt{ } x}\right),
\end{aligned}
$$


where $|A|$ is limited for all positive values of $x$, we have

$$
\begin{aligned}
& \frac{\Pi(n+r)}{\Pi\left(n+r+\frac{1}{2}\right)} \\
& \quad=e^{\frac{1}{5}} \frac{(n+r)^{\frac{1}{2}}}{n+r+\frac{1}{2}}\left[1+\frac{1}{2(n+r)}\right]^{-(n+r)}\left(1+\frac{A}{\sqrt{n+r}}\right)\left(1+\frac{A^{\prime}}{\sqrt{n+r+\frac{1}{2}}}\right)^{-1},
\end{aligned}
$$

where $|A|,\left|A^{\prime}\right|$ are limited for all values of $n$ and $r$.

We have

$$
\frac{A^{\prime}}{\sqrt{n+r+\frac{1}{2}}}=\frac{L^{\prime \prime}}{\sqrt{n+r}} .
$$

where $\left|A^{\prime \prime}\right|$ is limited, and $\left(1+\frac{A^{\prime \prime}}{\sqrt{ } n+r}\right)^{-1}$ lies between $1-\frac{A^{\prime \prime}}{\sqrt{n+r}}$ and 1 , for all sufficiently large values of $n+r$; therefore

$$
\left(1+\frac{A^{\prime}}{\sqrt{n}+r^{\prime}+\frac{i}{2}}\right)^{-1}=1+\frac{A^{\prime \prime \prime}}{\sqrt{ } n+r},
$$

where $\left|A^{\prime \prime \prime}\right|$ is limited for all values of $n$ and $r$. Again, we have

$$
\left(1+\frac{A}{\sqrt{n+r}}\right)\left(1+\frac{A^{\prime \prime \prime}}{\sqrt{n+r}}\right)=1+\frac{1}{\sqrt{n+r}}\left(A+A^{\prime \prime \prime}+\frac{A A^{\prime \prime \prime}}{n+r}\right)=1+\frac{B}{\sqrt{n+r}},
$$

where $|B|$ is limited for all values of $n$ and $r$.

The expression

$$
\frac{e^{h}}{\sqrt{ } n+r}\left[1+\frac{1}{2(n+r)}\right]^{-(n+r+1)}\left(1+\frac{B}{\sqrt{ } n+r}\right)
$$

is equivalent to

$$
\frac{e^{\frac{1}{2}}}{\sqrt{n} \overline{+r}}\left[1+\frac{1}{2(n+r)}\right] e^{-x \log (1+1 / 2 x)}\left(1+\frac{B}{\sqrt{ } n+\bar{r}}\right)
$$

where $x=n+r$. Also $e^{-\lrcorner \log (1+1 / 2 x)}=e^{-x\left(1 / 2 x-\theta, 8 x^{2}\right)}$,

where $0<\theta<1$, and this is equivalent to $e^{\frac{1}{s}}\left(1+\frac{\alpha}{x}\right)$, where $\alpha$ is limited for all positive values of $x$ greater than 1 . It now follows that

$$
\begin{aligned}
\frac{\Pi(n+r)}{\Pi\left(n+r+\frac{1}{2}\right)} & =\frac{1}{\sqrt{n+r}}\left(1+\frac{1}{2(n+r) j}\left(1+\frac{a}{n+r}\right)\left(1+\frac{B}{\sqrt{n+r}}\right)\right. \\
& =\frac{1}{\sqrt{n+r}}\left(1+\frac{\gamma}{\sqrt{n+r}}\right),
\end{aligned}
$$

when $|\gamma|$ is less than some tixed positive number, for all values of $n$ und $r(>0)$. 
We have now obtained for $P_{n}(\cos \theta)$ the expression

$$
\begin{aligned}
\frac{2}{\sqrt{ } \pi}\left[\left(\frac{1}{n^{\frac{1}{2}}}+\frac{\gamma}{n^{\frac{2}{3}}}\right)\right. & \sin (n+1) \theta \\
& \left.+\sum_{r=1}^{\infty} \frac{1.3 \ldots 2 r-1}{2.4 \ldots 2 r} ! \frac{1}{(n+r)^{\frac{1}{2}}}+\frac{\gamma}{(n+r)^{\frac{2}{3}}} ; \sin (n+2 r+1) \theta\right],
\end{aligned}
$$

where $\gamma$ depends on $n$ and $r$, but is numerically less than some fixed number independent of $n, r$, and $\theta$. This holds for every value of $\theta$ except for $\theta=0$ and $\theta=\pi$.

To sum the series

$$
\frac{1}{n^{\frac{1}{2}}} \sin (n+1) \theta+\sum_{r=1}^{\infty} \frac{1.3 \ldots 2 r-1}{2.4 \ldots 2 r} \frac{1}{(n+r)^{\frac{3}{2}}} \sin (n+2 r+1) \theta,
$$

we observe that, if $|h|<1$,

$$
e^{(n+1) t \theta}\left(1-h \cdot e^{2 \cdot \theta}\right)^{-\frac{1}{2}}=e^{(n+1) t \theta}+\sum_{i=1}^{\infty} \frac{1.3 \ldots 2 r-1}{2.4 \ldots 2 r} h^{r} e^{(n+2 r+1) t \theta},
$$

with a similar equality in case the sign of $\iota$ is changed. Writing $h=e^{-u^{2}}$, we deduce that

$$
\begin{aligned}
& \frac{1}{2 \iota} e^{-n u^{u}}\left\{e^{(n+1) \iota \theta}\left(1-e^{-u^{2}} e^{2 \iota \theta}\right)^{-t}-e^{-(n+1) \iota \theta}\left(1-e^{-u^{2}} e^{-2 \iota t}\right)^{-t+t}\right\} \\
& =e^{-n u^{2}} \sin (n+1) \theta+\sum_{r=1}^{n} \frac{1.3 .5 \ldots 2 r-1}{2.4 .6 \ldots 2 r} e^{-(n+r) x^{2}} \sin (n+2 r+1) \theta,
\end{aligned}
$$

where $u>0$.

We now employ the known theorem* that, if $\Sigma a_{r}$ be any convergent series, and if $u_{1}(x), u_{2}(x), \ldots, u_{r}(x), \ldots$ be defined for the interval $(\alpha, \beta)$ of $x$, and be limited and positive in that interval, and such that $u_{r}(x) \geqslant u_{r+1}(x)$ for every value of $r$ and $x$, then $\Sigma a_{n} u_{n}(x)$ converges uniformly in the interval $(\alpha, \beta)$. Since $e^{-r u^{2}} \geqslant e^{-(r+1) u^{*}}$ for all values of $u$ in any interval $(0, \lambda)$, we see that the above series converges uniformly for all values of $u$ in such interval. It follows that the above series may be integrated term by term, between the limits 0 and $\lambda$ of $u$, and that the series so obtained converges to the integral of the sum of the above series. In order to shew that we may integrate term by term with respect to $u$ from 0 to $\infty$, it is sufficient $\dagger$ to shew that the additional condition is satisfied that $\lambda$ and $r_{1}$ can be so chosen, corresponding to an arbitrarily chosen positive number $\epsilon$, that

$$
\int_{\lambda}^{\lambda}\left[e^{-n u^{2}} \sin (n+1) \theta+\sum_{s=1}^{s=1} \frac{1.3 .5 \ldots 2 s-1}{2.4 .6 \ldots 2 s} e^{-(n+s) u^{2}} \sin (n+2 s+1) \theta\right] d u
$$


is numerically less than $\epsilon$, for every value of $\lambda^{\prime}>\lambda$, and for every value of $r \geqslant r_{1}$. This expression is clearly less numerically than

$$
\int_{\lambda}^{\lambda^{\prime}}\left[e^{-u u^{2}}+\sum_{s=1}^{s=r} e^{-(n+s) u^{2}}\right] d u
$$

or than $\int_{\lambda}^{\lambda} \frac{e^{-n \prime^{2}}}{1-e^{-u^{2}}} d u$, and this is less than $\frac{1}{1-e^{-1}} \int_{\lambda}^{\infty} e^{-n u^{2}} d u$, if $\lambda>1$.

Hence, since $\int_{\lambda}^{\infty} e^{-\imath u^{2}} d u$ is convergent, it is clear that $\lambda$ can be so chosen that, for every value of $r$, the above expression is numerically less than $\epsilon$. Remumbering that

$$
\int_{0}^{\infty} e^{-(n+r) u^{2}} d u=\frac{\sqrt{ } \pi}{2 \sqrt{ }(n+r)},
$$

we now tind that

$$
\begin{aligned}
& \frac{\sqrt{ } \pi}{2} \cdot \frac{1}{n^{\frac{1}{2}}} \sin (n+1) \theta+\sum_{r=1}^{\infty} \frac{1.3 \ldots 2 r-1}{2.4 \ldots 2 r} \frac{1}{(n+r)^{\frac{1}{2}}} \sin (n+2 r+1) \theta^{!}
\end{aligned}
$$

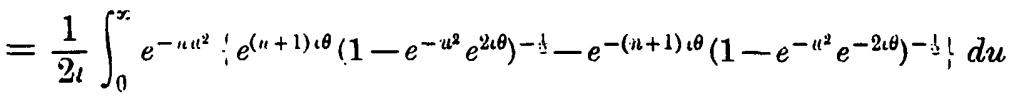

$$
\begin{aligned}
& =\frac{1}{2 \imath n^{4}} \int_{0}^{y} e^{-\iota^{2}}\left[e^{(n+1) \iota \theta}\left(1-e^{-v^{2} n} e^{2 \imath \theta}\right)^{-1}-e^{-(n+1) \iota \theta}\left(1-e^{-v^{2} i n} e^{-2 \iota \theta}\right)^{-t}\right] d v .
\end{aligned}
$$

Writing $1-e^{-r^{2} i t} \cos 2 \theta=K \cos \phi, e^{-r " n} \sin 2 \theta=R \sin \phi$, the expression on the right-hand side becomes $\frac{1}{n^{\frac{1}{2}}} \int_{0}^{\infty} \frac{e^{-v^{2}}}{R^{\frac{1}{2}}} \sin \left\{(n+1) \theta+\frac{1}{2} \phi\right\} d v$.

It is now seen that

$$
\frac{2}{\sqrt{ } \pi}\left[\frac{1}{n^{n}} \sin (n+1) \theta+\sum_{r=1}^{\infty} \frac{1.3 \ldots 2 r-1}{2 \cdot 4 \ldots 2 r} \frac{1}{(n+r)^{\frac{3}{3}}} \sin (n+2 r+1) \theta\right]
$$

is numerically less than $\frac{ \pm}{\pi} \frac{1}{n^{\frac{1}{2}}} \int_{0}^{\infty} \frac{1}{R^{\frac{1}{2}}} e^{-v^{2}} d u$.

$$
\text { Also } \quad \frac{\sin ^{2} \theta}{R^{2}}=\frac{\sin ^{2} \theta}{\left(1-e^{-v^{2} / n}\right)^{2}+4 e^{-v^{2} / n} \sin ^{2} \theta} \leqslant \frac{1}{4 e^{-v^{2} / n}} \text {; }
$$

hence, choosing a number o between 0 and 1 , we see that $\frac{\sin ^{2} \theta}{R^{2}} \leqslant \frac{1}{4 \delta}$, if $e^{-v^{2} / n}$ is between 1 and $\delta$. If $e^{-v^{2} / n}$ lies between $\delta$ and 0 , we see that

$$
\frac{\sin ^{2} \theta}{R^{2}}<\frac{\sin ^{2} \theta}{\left(1-e^{-c^{2} / n}\right)^{2}}<\frac{\sin ^{2} \theta}{(1-\delta)^{2}}, \quad \text { or } \quad \frac{\sin ^{2} \theta}{R^{2}}<\frac{1}{(1-\delta)^{2}}
$$


It thus appears that $\frac{\sin ^{2} \theta}{R^{2}}$ is less than some fixed positive number $K^{\prime}$, for every value of $\theta$ and $\eta$. Therefore

$\sqrt{n} \overline{\sin } \bar{\theta} \frac{2}{\sqrt{ } \pi}\left[\frac{1}{n^{\frac{2}{2}}} \sin (n+1) \theta+\sum_{r=1}^{\infty} \frac{1.3 \ldots 2 r-1}{2.4 \ldots 2 r} \frac{1}{(n+r)^{\frac{1}{2}}} \sin (n+2 r+1) \theta\right]$

is numerically less than a fixed positive number independent of $n$ and $\theta$; for

$$
\int_{0}^{\infty}\left(\frac{\sin \theta}{R}\right)^{\frac{1}{2}} e^{-v^{2}} d v<K^{\ddagger} \int_{0}^{\infty} e^{-v^{2}} d v<\frac{\sqrt{ } \pi}{2} K^{\ddagger} .
$$

We have next to consider the expression

$$
\frac{2}{\sqrt{ } \pi}\left[\frac{\gamma}{n^{\frac{3}{3}}} \sin (n+1) \theta+\sum_{r=1}^{\infty} \frac{1.3 \ldots 2 r-1}{2.4 \ldots 2 r} \frac{\gamma}{(n+r)^{\frac{2}{2}}} \sin (n+2 r+1) \theta\right],
$$

where the numbers $\gamma$ are less than some fixed number $A$ independent of $n, r$, and $\theta$. This expression is numerically less than

$$
\frac{2 A}{\sqrt{ } \pi}\left[\frac{1}{n^{\frac{\pi}{2}}}+\sum_{r=1}^{\infty} \frac{1.3 \ldots 2 r-1}{2.4 \ldots 2 r} \frac{1}{(n+r)^{\frac{3}{2}}}\right]
$$

or than $\frac{2 A}{\sqrt{ } \pi}\left[\frac{1}{n^{\frac{3}{3}}}+\int_{0}^{1} \frac{1}{(n+x)^{\frac{2}{2}}} d x+\int_{1}^{2} \frac{1}{(n+x)^{\frac{7}{3}}} d x+\ldots\right]$,

which is $\frac{2 A}{\sqrt{ } \pi}\left(\frac{1}{n^{\frac{2}{2}}}+\int_{0}^{\infty} \frac{1}{(n+x)^{\frac{3}{2}}} d x\right)$ or $\frac{2 A}{\sqrt{\pi}}\left(\frac{1}{n^{\frac{3}{2}}}+\frac{1}{2 n^{\frac{3}{3}}}\right)$.

If the expression be multiplied by $(n \sin \theta)^{\frac{1}{2}}$, we see that it is then numerically less than $\frac{2 A}{\sqrt{ } \pi}\left(\frac{1}{2}+\frac{1}{n}\right)$, or than $\frac{3 A}{\sqrt{ } \pi}$, if $n \geqslant 1$.

It has now been proved that $(u \sin \theta)^{ \pm} P_{n}(\cos \theta)$ is numerically less than some fixed positive number, independent of $n$ and $\theta$. This has been proved, on the assumption that $0<\theta<\pi$; but as the expression vanishes when $\theta=0$, or $\theta=\pi$, the result clearly holds for $0 \leqslant \theta \leqslant \pi$.

If $0<\theta<\pi$, and if $n$ and $\theta$ vary in such a way that $n \theta$ becomes indefinitely great, it follows from the above result that $P_{n}(\cos \theta)$ tends to the limit zero. This result has been otherwise established by Bruns.* It is well known that, if, as $n$ increases indefinitely, $n \theta$ tends to a finite limit $\rho$, then

$$
\lim _{n=\infty} P_{n}(\cos \theta)=J_{0}(\rho) .
$$

- Crelle's Journal, Vol. xc., p. 322. See also Heine's Kugelfunctionen, Vol. II., pp. 361-363. 
2. The sum of the first $n+1$ terms of the series for the representation of a function $f(x)$ by means of Legendre's functions is*

$$
\int_{-1}^{1} \frac{n+1}{2} P_{n+1} \frac{(x) P_{n}\left(x^{\prime}\right)-P_{n}(x) P_{n+1}\left(x^{\prime}\right)}{x-x^{\prime}} f\left(x^{\prime}\right) d x^{\prime} .
$$

We have here to consider the values of

$$
\int_{-1}^{-1+\varepsilon} \frac{n+1}{2} \frac{P_{n+1}(x)}{P_{n}\left(x^{\prime}\right)-P_{n}(x) P_{n+1}\left(x^{\prime}\right)} \frac{r^{\prime}}{r-r^{\prime}} f\left(x^{\prime}\right) d x^{\prime},
$$

with the similar integral taken between the limits $1-\epsilon$ and 1. The point $x$ is taken to be interior to the interval $(-1,1)$. We may assume $\epsilon$ to be so small that $x+1-\epsilon \geqslant \mu$, where $\mu$ is a fixed positive number.

We have

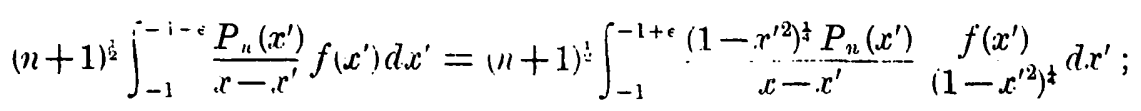

now let it be assumed that $\frac{f\left(x^{\prime}\right)}{\left(1-x^{\prime 2}\right)^{2}}$ has a Lebesgue integral in the whole interval $(-1,1)$ : then the above expression is numerically less than

$$
\frac{k}{\mu} \int_{-1}^{-1+\epsilon}\left|\frac{f\left(x^{\prime}\right)}{\left(1-x^{\prime 2}\right)^{\frac{1}{2}}}\right| d x^{\prime},
$$

where $k$ is a positive number, such that

$$
(n+1)^{\frac{1}{2}}\left(1-x^{\prime 2}\right)^{\frac{1}{2}} P_{n}\left(x^{\prime}\right)<k,
$$

for all values of $n$ and $x^{\prime}$, in uccordance with the theorem established in \$1. It follows that $\epsilon$ may be so chosen that the numerical value of

$$
(n+1)^{\frac{1}{s}} \int_{-1}^{-1+\epsilon} \frac{P_{n}\left(x^{\prime}\right)}{r-x^{\prime}} f\left(x^{\prime}\right) d x x^{\prime}
$$

is less than an arbitrarily chosen positive number, for all values of $n$, and for all values of $x$ in an interval interior to $(-1,1)$.

Since $(n+1) ! P_{n+1}(x)$ is numerically less than a fixed positive number, for all points $x$ in such an interval, we see that $\epsilon$ can be so chosen that

$$
\int_{-1}^{-1+e} \frac{n+1}{2} \frac{P_{n+1}(x) P_{n}\left(x^{\prime}\right)}{x-x^{\prime}} f\left(x^{\prime}\right) d x^{\prime}
$$

is, in numerical value, less than an arbitrarily chosen positive number $\xi$. 
As the same argument applies to

$$
\int_{-1}^{-1+e} \frac{n+1}{2} \frac{P_{n}(x) P_{n+1}\left(x^{\prime}\right)}{x-x^{\prime}} d x^{\prime}
$$

we see that $\epsilon$ can be so chosen that

$$
\left|\int_{-1}^{-1+e} \frac{n+1}{2} P_{n+1} \frac{(x) P_{n}\left(x^{\prime}\right)-P_{n}(x) P_{n+1}\left(x^{\prime}\right)}{x-x^{\prime}} f\left(x^{\prime}\right) d x x^{\prime}\right|<\xi
$$

for all values of $n$, and for all values of $x$ in a prescribed interval interior to $(-1,1)$. A similar proof applies to the case when the limits of integration are $1-\epsilon$ and 1 .

Let $f_{1}(x)$ be a function such that $f_{1}(x)=f^{\prime}(x)$ in the interval $(-1+\epsilon$, $1-\epsilon)$, and such that $f_{1}(x)=0$ when $-1 \leqslant x<-1+\epsilon$, and when $1-\epsilon<x \leqslant 1$. Since the function $f_{1}(x)$ is of limited total fluctuation in the neighbourhoods of the points $-1,+1$, the result obtained in my earlier paper (loc. cit., p. 395) is applicable to this function. Therefore

$$
\int_{-1+c}^{1-e} \frac{n+1}{2} \frac{P_{n+1}(x) P_{n}\left(x^{\prime}\right)-P_{n}(x) P_{n+1}\left(x^{\prime}\right)}{x-x^{\prime}} f\left(x^{\prime}\right) d x^{\prime}
$$

differs from $\frac{1}{2}\{f(x+0)+f(x-0)$ \} by less than $\bar{\zeta}$, provided $n$ is not less than some fixed integer $n_{1}$, and provided $x$ is a point in the interval $(-1+\epsilon+\mu, 1-\epsilon-\mu)$ for which point a neighbourhood exists in which the function is of limited total fluctuation.

It now follows that

$$
\int_{-1}^{1} \frac{n+1}{2} \frac{P_{n+1}(x) P_{n}\left(x^{\prime}\right)-P_{n}(r) P_{n+1}\left(x^{\prime}\right)}{x-r^{\prime}} f\left(. r^{\prime}\right) d \cdot x^{\prime}
$$

differs from $\frac{1}{2}\left\{f(x+0)+f(x-0)\right.$; ly less than $3 \dot{\zeta}$, for $n \geqslant n_{1}$, where $x$ satisfies the condition just stated.

The following theorem has now been established :-

Let $f(x)$ be a function [limited or unlimiter in (-1. 1)] such that $\frac{f^{\prime}(x)}{\left(1-x^{2}\right)^{\frac{1}{2}}}$ has a Lebesgue integral in the interval $(-1,+1)$. It is sufficient for the convergence of the series

$$
\sum_{n=0} \frac{2 n+1}{2} P_{n}(x) \int_{-:}^{1} f\left(x^{\prime}\right) P_{n}\left(x^{\prime}\right) d x^{\prime}
$$

to the value $\frac{1}{2}\left\{f^{\prime}(x+0)+f(x-0)\right\}$, at any point $x$ in the interior of the interval $(-1,1)$, that a neighbourhood of the point $x$ should exist in which $f(x)$ is of limited total fluctuation.

In any interval in which $f(x)$. is continuous, and which is contained. 
in the intring of another interval in which the function has limited total thuctuation, the convergence of the series to the value of $f(x)$ is uniform.

The condition that $\frac{f(x)}{\left(1-x^{2}\right)^{\frac{3}{4}}}$ should have a Lebesgue integral is equivalent to the conditions that $f(x)$ should have a Lebesgue integral in $(-1,1)$, and that the two Lebesgue integrals $\int_{-1}^{-1+\eta} \frac{f(x)}{(1+x)^{\frac{1}{4}}} d x$, $\int_{1-\eta}^{1} \frac{f(x)}{(1-x)^{\frac{1}{2}}} d x$ should also exist.

In case $|f(x)|$ has tinite upper limits in neighbourhoods of the points $1,-1$, the existence of $\int_{-1}^{1} f(x) d x$ is sufficient to ensure that $\int_{-1}^{1} \frac{f(x)}{\left(1-x^{2}\right)^{\frac{1}{4}}} d x$ also exists.

More generally, it is sufficient that $f(x)$ should have a Lebesgue integral in $(-1,1)$, and that $|f(x)|<\frac{A}{(1+x)^{k}}$ in a neighbourhood of the point -1 , and $|f(x)|<\frac{A^{\prime}}{(1-x)^{k}}$ in a neighbourhood of the point 1 , where $k$ and $k^{\prime}$ are each less than $\frac{3}{4}$, and $A, A^{\prime}$ are positive numbers. The known logarithmic conditions for the convergence of an integral in the neighbourhood of a singularity are also sufficient.

A particular case of this theorem has been given by Darboux, * who shewed that, if $f(x)$ has the values $\frac{A}{(1+x)^{k}}, \frac{A^{\prime}}{(1-x)^{k^{\prime}}}$, where $k<\frac{3}{4}$, $k^{\prime}<\frac{3}{4}$, in neighbourhoods of the points $-1,1$, the series is so far convergent.

It may be observed that the above general theorem may be proved directly by employing the general convergence theorem (loc. cit., p. 350), and proceeding as in my earlier paper.

For this purpose we take

$$
F\left(x^{\prime}, x, n\right)=\frac{n+1}{2}\left(1-x^{\prime 2}\right)^{\sharp} \frac{P_{n+1}(x) P_{n}\left(x^{\prime}\right)-P_{n}(x) P_{n+1}\left(x^{\prime}\right)}{x-x^{\prime}},
$$

and verify that $\left|F\left(x^{\prime}, x, n\right)\right|$ is less than a fixed positive number for all values of $x^{\prime}$ in the interval $(-i, 1)$, for all values of $x$ in an interval $(-1+\epsilon, 1-\epsilon)$, and such that $\left|x-x^{\prime}\right| \geqslant \mu$, and for all values of $n$. It can then be verified, as before (loc. cit., pp. 389,390 ) that

$$
\left|\int_{a_{1}}^{\beta_{1}} F\left(x^{\prime}, x, n\right) d x^{\prime}\right|<\frac{1}{\mu n^{3}}|\alpha(n)|,
$$

* See Liouville's Journal, Ser. 3, Vol. Iv., p. 393. 
only i slight modification of method being necessary on account of the existence of the factor $\left(1-x^{\prime 2}\right)^{\frac{1}{7}}$. In the rest of the work, the function $f(x)$ must then be replaced by $\frac{f(x)}{\left(1-x^{2}\right)^{\frac{3}{2}}}$, and the convergence of

$$
\frac{n+1}{2} \int_{-1}^{1} \frac{P_{n+1}(x) P_{n}\left(x^{\prime}\right)-P_{n}(x) P_{n+1}\left(x^{\prime}\right)}{x-x^{\prime}} f\left(x^{\prime}\right) d x^{\prime}
$$

is then established, subject to the conditions stated in the theorem.

3. It will now be shewn that, if in the neighbourhood of the point $-1, f\left(x^{\prime}\right)$ is of the form $\frac{A}{\left(1+x^{\prime}\right)^{k}}+f_{1}\left(x^{\prime}\right)$, where $k \geqslant \frac{3}{4}$, and $f_{1}\left(x^{\prime}\right)$ is limited in the neighbourhood, then the series does not converge at the point $x$ interior to the interval $(-1,1)$. It is clear that, in a sufficiently small neighbourhood of the point $-1, \frac{f\left(x^{\prime}\right)}{x-x^{\prime}}$ is of the form

$$
\frac{B}{\left(1+x^{\prime}\right)^{\prime:}}+f_{2}\left(x^{\prime}\right) \text {, }
$$

where $f_{2}\left(x^{\prime}\right)$ is limited in the neighbourhood.

It will be sufficient to prove that

$$
\int_{-1}^{1} \frac{n^{\frac{1}{3}} P_{n}\left(x^{\prime}\right)}{\left(1+x^{\prime}\right)^{k}} d x^{\prime}
$$

does not converge to a definite limit, when $n$ is indefinitely increased, provided $k \geqslant \frac{3}{4}$; the condition that $k<1$ is necessary for the existence of the integrel. For it then follows that $\int_{-1}^{-1+e} \frac{n^{\frac{1}{3}} P_{n}\left(x^{\prime}\right)}{\left(1+x^{\prime}\right)^{k}} d x$ does not converge to a definite limit, when $n$ is indefinitely increased, and therefore the same holds of

$$
\frac{n+1}{2} \int_{-1}^{1} \frac{P_{n+1}(x) P_{n}\left(x^{\prime}\right)}{\left(1+x^{\prime}\right)^{k}} d x^{\prime} .
$$

We have $P_{n}\left(x^{\prime}\right)=A_{0}+A_{1}\left(\frac{1+x^{\prime}}{2}\right)+A_{2}\left(\begin{array}{c}1+x^{\prime} \\ 2\end{array}\right)^{2}+\ldots+A_{\text {" }}\left(\frac{1+x^{\prime}}{2}\right)^{n}$, where $\quad A_{0}+A_{1}+\ldots+A_{n}=1$.

Hence, we find that

$$
\begin{aligned}
n^{3} \int_{-1}^{1} \frac{P_{n}\left(x^{\prime}\right)}{\left(1+x^{\prime}\right)^{k}} d x^{\prime} & =n^{\frac{1}{b}} \cdot 2^{1-k}\left[\frac{A_{0}}{1-k}+\frac{A_{1}}{2-k}+\ldots+\frac{A_{n}}{n+1-k}\right] \\
& =n^{\frac{1}{b}} \cdot 2^{1-k}(-1)^{n} \frac{k(k+1) \ldots(k+n-1)}{(1-k)(2-\bar{k}) \ldots(n+1-k)},
\end{aligned}
$$

SER. 2. VOL. T. NO. 1007. 
since the integral vanishes for the values $0,-1,-2, \ldots-(n-1)$, of $k$. The expression on the right-hand side may be written in the form

$$
(-1)^{n} n^{2} \cdot 2^{1-k} \frac{\Pi(k+n-1)}{\Pi(k-1)} \frac{\Pi(-k)}{\Pi(n+1-k)} ;
$$

and the asymptotic value of this is $(-1)^{n} e^{-2 k+2} \cdot 2^{1-k} \frac{\Pi(-k)}{\Pi(k-1)} n^{2(k-1)+k}$. If $k>\frac{3}{4}$, this increases indefinitely as $n$ does so; and if $k=\frac{3}{4}$, it has no determinate value. If $k<\frac{3}{4}$, it converges to zero, when $n$ is indefinitely increased.

It therefore appears that, if the function $f(x)$ is of the form

$$
\frac{A}{(1+x)^{k}}+f_{1}(x)
$$

in the neighbourhood of the point -1 , where $k \geqslant \frac{3}{4}$, then the series does not converge at any point $x$ interior to the interval $(-1,1)$.

That the convergence of the series may be, throughout the interval, clestroyed by the effect of the values of the function in the neighbourhoods of the points $-1,1$, is illustrated by this result.*

4. There remains the consideration of the convergence of the series at the end-points of the interval $(-1,1)$. This is a matter of some importance, because, in accordance with the usual method of investigating the convergence of the expansion of a function in spherical surface harmonics, the convergence of the expansion of a certain subsidiary function in a series of Legendre's functions at one of the ends of the interval of representation is decisive in relation to the convergence of the series of surface harmonics.

It appears that, if $f(x)$ be a function which possesses a Lebesgue integral in the inte:val $(-1,1)$, the existence of neighbourhoods of the points $-1,1$ in which $f(x)$ is of limited total fluctuation is not sufficient to ensure that the series

$$
\sum_{n=0} \frac{2 n+1}{2} P_{n}(x) \int_{-1}^{1} f\left(x^{\prime}\right) P_{n}\left(x^{\prime}\right) d x^{\prime}
$$

converges at those points. It is however sufficient, although not necessary, that $f(x)$ should be of limited total fluctuation in the whole interval $(-1,1)$.

- This result was given by Darboux. See Liouville's Journal, Ser. 3, Vol. virr., p. 393. 
Let us consider the series at the point $x=1$; the sum of the first $2 n+1$ terms is

$$
\frac{n+1}{2} \int_{-1}^{1} \frac{P_{n}\left(x^{\prime}\right)-P_{n+1}\left(x^{\prime}\right)}{1-x^{\prime}} f\left(x^{\prime}\right) d x^{\prime} .
$$

We shall first estimate the limit, when $n$ is indefinitely increased, of

$$
\frac{n+1}{2} \int_{a}^{\beta} \frac{P_{n}\left(x^{\prime}\right)-P_{n+1}\left(x^{\prime}\right)}{1-x^{\prime}} f\left(x^{\prime}\right) d x^{\prime},
$$

where

$$
-1<\alpha<\beta<1 \text {. }
$$

On substitution of the expressions for $P_{n}\left(x^{\prime}\right), P_{n+1}\left(x^{\prime}\right)$ given in $\$ 1$, and which are valid for the interval $(a, \beta)$ of $x^{\prime}$, it is easily seen that the limit is the same as that of

$$
\begin{aligned}
\frac{n^{\frac{3}{2}}}{2}\left(\frac{2}{\pi}\right)^{\frac{3}{3}} \int_{a}^{\beta}\left[\operatorname { c o s } \left\{\left(n+\frac{1}{2}\right)\right.\right. & \left.\theta^{\prime}-\frac{\pi}{4}\right\} \\
& \left.-\cos \left\{\left(n+\frac{3}{2}\right) \theta^{\prime}-\frac{\pi}{4}\right\}\right]\left(\sin \theta^{\prime}\right)^{-\frac{3}{3}} \frac{f\left(x^{\prime}\right)}{1-x^{\prime}} d x^{\prime},
\end{aligned}
$$

where $x^{\prime}=\cos \theta^{\prime}$; and this is of the form

$$
n^{3}\left(\frac{2}{\pi}\right)^{3} \int_{p}^{4} \sin \left\{(n+1) \theta^{\prime}-\frac{\pi}{4}\right\} F\left(\theta^{\prime}\right) d \theta^{\prime},
$$

where $0<p<q<\pi$.

If $f\left(x^{\prime}\right)$ have a Lehesgue integral in the interval $(\alpha, \beta)$, then $F\left(\theta^{\prime}\right)$ has a Lebesgue integral in the interval $(p, q)$. It then follows, from it corollary to the fundamental convergence theorem given in my earlier paper (p. 355), that

$$
\lim _{n=\infty} \int_{p}^{4} \sin \left\{(n+1) \theta^{\prime}-\frac{\pi}{4}\right\} F\left(\theta^{\prime}\right) d \theta^{\prime}=0,
$$

but it does not necessarily follow that

$$
\lim _{n=\infty} n^{\frac{1}{2}} \int_{p}^{q} \sin \left\{(n+1) \theta^{\prime}-\frac{\pi}{4}\right\} F\left(\theta^{\prime}\right) d \theta^{\prime}=0 .
$$

If, however, $f\left(x^{\prime}\right)$ is of limited total fluctuation in the interval $(\alpha, \beta)$, in which case $F\left(\theta^{\prime}\right)$ is of limited total fluctuation in $(p, q)$, the latter equality holds. To see this we may take $F\left(\theta^{\prime}\right)$ to be the difference of two functions $F_{1}\left(\theta^{\prime}\right), F_{2}\left(\theta^{\prime}\right)$, each of which is monotone in $(p, q)$. We have then $\int_{p}^{q} \sin \left\{(n+1) \theta^{\prime}-\frac{\pi}{4}\right\} F_{1}\left(\theta^{\prime}\right) d \theta^{\prime}$

$=F_{1}(p+0) \int_{p}^{\lambda} \sin \left\{(n+1) \theta^{\prime}-\frac{\pi}{4}\right\} d \theta^{\prime}+F_{1}(q-0) \int_{\lambda}^{Q} \sin \left\{(n+1) \theta^{\prime}-\frac{\pi}{4}\right\} d \theta^{\prime}$, 
where $\lambda$ is a number such that $p \leqslant \lambda \leqslant q$. The integral on the lefthand side is consequently not greater, in absolute value, than

$$
\frac{2}{n+1}\left\{\left|F_{1}(p+0)\right|+\left|F_{1}(q-0)\right|\right\} \text {. }
$$

It therefore follows that

$$
\lim _{n=\pi} n^{2} \int_{1}^{\prime \prime} \sin i_{i}^{\prime}(n+1) \theta^{\prime}-\frac{\pi}{t} !_{i} F_{1}\left(\theta^{\prime}\right) d \theta^{\prime}=0 .
$$

We may clearly substitute $F_{2}\left(\theta^{\prime}\right)$, and therefore also $F\left(\theta^{\prime}\right)$ in this result. It follows that, if $f\left(x^{\prime}\right)$ have limited total fluctuation in $(\alpha, \beta)$, then

$$
\lim _{n=\infty} \frac{n+1}{2} \int_{a}^{\beta} \frac{P_{n}\left(x^{\prime}\right)-P_{n+1}\left(x^{\prime}\right)}{1-x^{\prime}} f\left(x^{\prime}\right) d: x^{\prime}=0 .
$$

Let us next suppose that, in the neighbourhood $(\xi-\eta, \xi+\eta)$ of a point $\xi$ in the interior of the interval $(\alpha, \beta), f(x)$ is of the form $\frac{A}{(x-\xi)^{k}}+\phi(x)$, where $0<k<1$, and $\phi(x)$ is of limited total fluctuation in the interval $(\hat{\xi}-\eta, \hat{\xi}+\eta)$. We may assume that in the intervals $(\alpha, \hat{\xi}-\eta),(\hat{\xi}+\eta, \beta)$ the function $f(x)$ is of limited total fluctuation; thus $f(x)$ has a single infinite discontinuity at the point $\xi$.

In this case, we see that in $(\xi-\eta, \xi+\eta)$ the function $F\left(\theta^{\prime}\right)$ is of the form $\frac{B}{\left(\theta^{\prime}-\gamma\right)^{k}}+F_{1}\left(\theta^{\prime}\right)$, where $\cos \gamma=\hat{\xi}$, and where $F_{1}\left(\theta^{\prime}\right)$ is of limited total fluctuation in $(\hat{\xi}-\eta, \hat{\xi}+\eta)$.

We then see that, to estimate the effect of the infinite discontinuity we have to evaluate

$$
\lim _{n=\infty} n^{k} \int_{\gamma-\eta_{1}}^{\gamma+\eta_{2}} \frac{B}{\left(\theta^{\prime}-\gamma\right)^{k}} \sin \left\{(n+1) \theta^{\prime}-\frac{\pi}{4}\right\} d \theta^{\prime}
$$

or

$$
\lim _{n=\infty} B n^{\frac{1}{2}} \int_{-\eta_{1}}^{\eta_{2}} \frac{1}{u^{k}} \sin \left\{(n+1) u+(n+1) \gamma-\frac{\pi}{4}\right\} d u \text {. }
$$

Writing $(n+1) u=v$, we see that the limit is dependent upon

$$
\lim _{n=\infty} n^{k-1} \int_{-(n+1) \eta_{1}}^{(n+1) \eta_{2}} \frac{\sin v}{v^{k}} d v \text { and } \lim _{n=-x} n^{k-1} \int_{-(n+1) \eta_{1}}^{(n+1) \eta_{2}} \frac{\cos v}{v^{k}} d v .
$$

If $k<\frac{1}{2}$, both these latter limits exist, and are zero. If $k=\frac{1}{2}$, the required limit has no definite value. In case $\frac{1}{2}<k<1$, the expression increases indefinitely as $n$ does so. 
It has now been shewn that, in case there is an infinite discontinuity of the function $f(x)$ at a point $\xi$, of order $\geqslant \frac{1}{2}$, it is impossible that

$$
\lim _{n=\infty} \frac{n+1}{2} \int_{a}^{\beta} \frac{P_{n}\left(x^{\prime}\right)-P_{n+1}\left(x^{\prime}\right)}{1-x^{\prime}} f^{\prime}\left(x^{\prime}\right) d x^{\prime}=0,
$$

it being assumed that the function is of limited total Huctuation, except in the neighbourhood of the point $\hat{\xi}$. In this case, although $f(x)$ may have a Lebesgue integral in the whole interval $(-1,1)$, and, although the function may have limited total fluctuation in neighbourhoods of the points $-1,1$, it is impossible that the series should converge at those points.

It may also happen that the above limit does not exist, and therefore that the series does not converge at the point 1 , when $f^{\prime}(x)$ is limited in $(\alpha, \beta)$ without being of limited total Huctuation. Therefore the series corresponding to a function which is limited in $(-1,1)$ and has a Lebesgue integral in that interval does not necessarily converge at the point 1.

For the purpose of considering those parts of the integral which represents the sum of the first $n+1$ terms of the series at $x=1$ which are in the neigbbourhood of the points $1,-1$, it is convenient to replace $\frac{n+1}{2} \frac{P_{n}\left(x^{\prime}\right)-P_{n+1}\left(x^{\prime}\right)}{1-x^{\prime}}$ by the equivalent expression

$$
\frac{1}{2}\left(\frac{d P_{n}\left(x^{\prime}\right)}{d x^{\prime}}+\frac{d P_{n+1}\left(x^{\prime}\right)}{d x^{\prime}}\right) \text {. }
$$

We have then to consider the integrals

$$
\begin{aligned}
& \frac{1}{2} \int_{-1}^{-1+e} ; \frac{d P_{n}\left(x^{\prime}\right)}{d x^{\prime}}+\frac{d P_{n+1}\left(x^{\prime}\right)}{d x^{\prime}} ; f\left(x^{\prime}\right) d x^{\prime}, \\
& \frac{1}{2} \int_{-1-e}^{1} ! \frac{d P_{n}\left(x^{\prime}\right)}{d x^{\prime}}+\frac{d P_{n+1}\left(x^{\prime}\right)}{d x^{\prime}} ; f\left(x^{\prime}\right) d x^{\prime} .
\end{aligned}
$$

Let it be assumed that $f\left(x^{\prime}\right)$ is monotone in each of the intervals $(-1,-1+\epsilon),(1-\epsilon, 1)$. The first integral is then equivalent to

$$
\begin{aligned}
& \frac{1}{2}\left\{P_{n}(-1+\mu)+P_{n+1}(-1+\mu)\right\}\{f(-1+0)-f(-1+\epsilon) \\
&+\frac{1}{2}\left\{P_{n}(-1+\epsilon)+P_{n+1}(-1+\epsilon)\right\} f(-1+\epsilon),
\end{aligned}
$$

where $\mu$ is such that $\theta \leqslant \mu \leqslant \epsilon$. This expression is numerically less than

$$
\bar{\zeta}+\frac{1}{2}\left|f(-1+\epsilon): P_{n}(-1+\epsilon)+P_{n+1}(-1+\epsilon) !\right|,
$$

if $\epsilon$ be so chosen that $|f(-1+0)-f(-1+\epsilon)|<\xi$. 
The number $\epsilon$ having been fixed, corresponding to an arbitrarily fixed positive number $\xi$, and also so that

$$
|f(1-0)-f(1-\epsilon)|<\xi
$$

we can determine a number $n_{1}$ such that

$$
\left|\frac{1}{2} \int_{-1}^{-1+\epsilon}\left\{\frac{d P_{n}\left(x^{\prime}\right)}{d x^{\prime}}+\frac{d P_{n+1}\left(x^{\prime}\right)}{d x^{\prime}}\right\} f\left(x^{\prime}\right) d x^{\prime}\right|<2 \xi,
$$

when $n \geqslant n_{1}$.

In a similar manner, we have

$$
\begin{aligned}
\frac{1}{2} \int_{1-\epsilon}^{1} i \frac{d P_{n}\left(x^{\prime}\right)}{d x^{\prime}}+\frac{d P_{n+1}\left(x^{\prime}\right)}{d x^{\prime}} ; f\left(x^{\prime}\right) d x^{\prime} \\
=f(1-0)+\frac{1}{2}\left\{P_{n}\left(1-\mu^{\prime}\right)+P_{n+1}\left(1-\mu^{\prime}\right)\right\}\{f(1-\epsilon)-f(1-0)\} \\
-\frac{1}{2} f(1-\epsilon)\left[P_{n}(1-\epsilon)+P_{n+1}(1-\epsilon)\right] .
\end{aligned}
$$

It then follows that a number $n_{2}$ can be so determined that

$$
\left|\frac{1}{2} \int_{1-\varepsilon}^{1}\left\lfloor\frac{d P_{n}\left(x^{\prime}\right)}{d x^{\prime}}+\frac{d P_{n+1}\left(x^{\prime}\right)}{d x^{\prime}}\right\} f\left(x^{\prime}\right) d x^{\prime}-f(1-0)\right|<2 \xi \text {, for } n \geqslant n_{2} .
$$

If now $f(x)$ satisfies sufficient conditions that

$$
\lim _{n=\infty} \int_{-1+e}^{1-\epsilon} \frac{n+1}{2} \frac{P_{n}\left(x^{\prime}\right)-P_{n+1}\left(x^{\prime}\right)}{1-x^{\prime}} f\left(x^{\prime}\right) d x^{\prime}=0,
$$

an integer $n_{3}$ can be determined such that

$$
\left|\int_{-1+e}^{1-e} \frac{n+1}{2} \frac{P_{n}\left(x^{\prime}\right)-P_{n+1}\left(x^{\prime}\right)}{1-x^{\prime}} f\left(x^{\prime}\right) d x^{\prime}\right|<\xi \text {, for } n \geqslant n_{3} .
$$

Let $n^{\prime}$ be the greatest of the three integers $n_{1}, n_{2}, n_{3}$; we have then

$$
\left|\int_{-1}^{1} \frac{n+1}{2} \frac{P_{n}\left(x^{\prime}\right)-P_{n+1}\left(x^{\prime}\right)}{1-x^{\prime}} f\left(x^{\prime}\right) d x^{\prime}-f(1-0)\right|<5 \xi,
$$

if $n \geqslant n^{\prime}$. Since $\xi$ is arbitrarily small, it has thus been shewn that, subject to the conditions assumed to be satisfied,

$$
\lim _{n=\infty} \int_{-1}^{1} \frac{n+1}{2} \frac{P_{n}\left(x^{\prime}\right)-P_{n+1}\left(x^{\prime}\right)}{1-x^{\prime}} f\left(x^{\prime}\right) d x^{\prime}=f(1-0) .
$$

The case of the convergence of the series at the point -1 may be treated in an exactly similar manner. 
The following theorem has now been established :-

Let $f(x)$ be a function which is of limited total fluctuation in neighbourhoods of the points $x=1, x=-1$. For the convergence of the series of Legendre's functions corresponding to $f(x)$, at the points $1,-1$ to the values $f(1-0), f(-1+0)$, it is insufficient that $f(x)$ have a Lebesgue integral in the vohole interval $(-1,1)$. It is, however, sufficient that $f(x)$ be of limited total fluctuation in $(-1,1)$. It is also sufficient that $f(x)$ be of limited total fluctuation when the neighbourhoods of a finite number of points in the interior of $(-1,1)$ are excluded, provided also that in each such neighbourhood of such a point $\dot{\xi}, f(x)$ is of the form $\frac{A}{\left(x-\xi^{k}\right)^{k}}+\phi(x)$, where $0<k<\frac{1}{2}$, and where $\phi(x)$ is of limited total fucctuation. In cuse, for such a point $\dot{\xi}, k \geqslant \frac{1}{2}$, the series does not converge to $f(1-0), f(-1+0)$ at the points $1,-1$. 